\title{
Applications of Asymmetric Hydrosilylations Mediated by Catalytic (DTBM-SEGPHOS)CuH
}

Bruce H. Lipshutz,** Asher Lower, Robert J. Kucejko, and Kevin Noson

${ }^{a}$ Department of Chemistry \& Biochemistry,

University of California, Santa Barbara, CA 93106 USA 


\section{Representative Procedure for the Asymmetric Hydrosilylation of Aryl Ketones using R-(-)-}

(DTBM-SEGPHOS)CuH: (R)-methyl-4-hydroxy-4-phenylbutanoate (2; Table 1, entry a).

To a flame-dried $10 \mathrm{~mL}$ round-bottom flask (RBF) equipped with a magnetic stir bar and purged with argon in a glovebox were added $\mathrm{CuCl}(1.5 \mathrm{mg}, 0.015 \mathrm{mmol}, 1.0 \mathrm{~mol} \%)$ and $\mathrm{NaO}-t-\mathrm{Bu}(1.4$ $\mathrm{mg}, 0.015 \mathrm{mmol}, 1.0 \mathrm{~mol} \%)$, (R)-(-)-DTBM-SEGPHOS (0.88 mg, $7.5 \times 10^{-4} \mathrm{mmol}, 0.05 \mathrm{~mol}$ $\%$, and $1.5 \mathrm{~mL}$ of toluene at $\mathrm{rt}$. The mixture was stirred for $30 \mathrm{~min}$ before being cooled to -78 ${ }^{\circ} \mathrm{C}$. PMHS $(0.39 \mathrm{~mL}, 6.0 \mathrm{mmol}, 4$ equiv $)$ was added to the RBF and the mixture was stirred for $15 \mathrm{~min}$. In a separate $10 \mathrm{~mL}$ pear-bottomed flask (PBF) were combined methyl-4-oxo-4phenylbutanoate $(0.26 \mathrm{~mL}, 1.50 \mathrm{mmol}), t-\mathrm{BuOH}(80 \mu \mathrm{L}, 1.50 \mathrm{mmol})$, and THF $(1.5 \mathrm{~mL})$ under argon, and the mixture was cooled to $-78^{\circ} \mathrm{C}$. The contents of the PBF were transferred via cannula to the RBF, and the reaction was monitored by TLC. Upon completion $(2 \mathrm{~h})$, the reaction was quenched with $2.5 \mathrm{M}$ aqueous $\mathrm{KF}(10 \mathrm{~mL})$ and $\mathrm{Et}_{2} \mathrm{O}(10 \mathrm{~mL})$ and stirred for $3 \mathrm{~h}$. The biphasic mixture was separated, and the aqueous phase was extracted with $\mathrm{Et}_{2} \mathrm{O}(3 \times 10$ $\mathrm{mL}$ ). The combined extracts were washed with brine and dried over anhydrous $\mathrm{MgSO}_{4}$. The solvent was removed under reduced pressure and the crude material was purified by silica gel chromatography (20\% EtOAc/hexanes) to yield $264 \mathrm{mg}(92 \%)$ of a colorless oil. Comparison of spectral data with known literature values confirmed the identity of the product. ${ }^{1}$ Chiral GC analysis (Chiraldex B-DM column, $140{ }^{\circ} \mathrm{C}$ ) indicated an ee of $99.4 \%$.

(R)-1-(Fluoro[2,3-c]pyridin-5-yl)ethanol (4; Table 1 entry b). The representative procedure was used with the following amounts of reagents: 1-(fluoro[2,3-c]pyridin-5-yl)ethanone (160 $\mathrm{mg}, 1.0 \mathrm{mmol}), \mathrm{CuCl}(2.0 \mathrm{mg}, 0.020 \mathrm{mmol}, 2.0 \mathrm{~mol} \%)$ and $\mathrm{NaO}-t-\mathrm{Bu}(1.9 \mathrm{mg}, 0.020 \mathrm{mmol}, 2.0$ mol \%), (R)-(-)-DTBM-SEGPHOS (3.0 mg, $\left.2.5 \times 10^{-3} \mathrm{mmol}, 0.25 \mathrm{~mol} \%\right), t$-BuOH (53 $\mu \mathrm{L}, 1.0$ mmol, 1.0 equiv), PMHS (0.26 mL, $4.0 \mathrm{mmol})$, THF $(1.0 \mathrm{~mL})$ and toluene $(1.0 \mathrm{~mL})$. The 
reaction was quenched with $2.5 \mathrm{M}$ aqueous $\mathrm{NaOH}$ rather than $\mathrm{KF}$. The title compound (141 mg, $88 \%$ ) was isolated as a colorless oil. Comparison of spectral data with known literature values confirmed the identity of the product. ${ }^{2}$ Chiral GC analysis (Chiraldex B-DM column, $80{ }^{\circ} \mathrm{C}$ ) indicated an ee of $97.1 \%$.

(R)-1-(3-(Trifluoromethyl)phenyl)ethanol (6; Table 1, entry c). The representative procedure was used with the following amounts of reagents: 3-trifluoromethylacetophenone (376 mg, 2.0 mmol), $\mathrm{CuCl}$ (4.0 mg, $0.04 \mathrm{mmol}, 2.0 \mathrm{~mol} \%$ ) and NaO-t-Bu (3.8 mg, $0.04 \mathrm{mmol}, 2.0 \mathrm{~mol} \%$ ), (R)-(-)-DTBM-SEGPHOS (3.0 mg, $\left.2.5 \times 10^{-4} \mathrm{mmol}, 0.25 \mathrm{~mol} \%\right), t$-BuOH (190 $\mu \mathrm{L}, 2.0 \mathrm{mmol}$, 1.0 equiv), PMHS $(130 \mu \mathrm{L}, 2.0 \mathrm{mmol})$, toluene $(2.0 \mathrm{~mL})$. The reaction was quenched with 2.5 $\mathrm{M}$ aqueous $\mathrm{NaOH}$. The title compound (354.2 $\mathrm{mg}, 95 \%)$ was isolated as a colorless oil. Comparison of spectral data with known literature values confirmed the identity of the product. ${ }^{3}$ Chiral GC analysis (Chiraldex BDM column, $80{ }^{\circ} \mathrm{C}$ ) indicated an ee of $95.3 \%$.

(R)-1-(3,5-bis(Trifluoromethyl)phenyl)ethanol (8; Table 1, entry d). The representative procedure was used with the following amounts of reagents: 3,5-bis(trifluoromethyl)acetophenone $(256.1 \mathrm{mg}, 1.0 \mathrm{mmol}), \mathrm{Cu}(\mathrm{OAc})_{2} \cdot \mathrm{H}_{2} \mathrm{O}(2.0 \mathrm{mg}, 0.01 \mathrm{mmol}, 1.0 \mathrm{~mol} \%), R-(-)-$ DTBM-SEGPHOS (2.9 mg, $\left.2.5 \times 10^{-4} \mathrm{mmol}, 0.25 \mathrm{~mol} \%\right), t$-BuOH $(80 \mu \mathrm{L}, 1.0 \mathrm{mmol}, 1.0$ equiv), PMHS (130 $\mu \mathrm{L}, 2.0 \mathrm{mmol})$, and toluene $(3.0 \mathrm{~mL})$. The reaction was quenched with 2.5 $\mathrm{M}$ aqueous $\mathrm{NaOH}$. The title compound (244 mg, 98\%) was isolated as a colorless oil. Comparison of spectral data with known literature values confirmed the identity of the product. ${ }^{4}$ Chiral GC analysis (Chiraldex B-DM column, $70{ }^{\circ} \mathrm{C}$ ) indicated an ee of $91.7 \%$.

(R)-3-Chloro-1-phenylpropan-1-ol (10; Table 1, entry e). The representative procedure was used with the following amounts of reagents: 3-chloro-1-phenylpropan-1-one (337 mg, 2.0 $\mathrm{mmol}), \mathrm{CuCl}(2.0 \mathrm{mg}, 0.020 \mathrm{mmol}, 2.0 \mathrm{~mol} \%)$ and $\mathrm{NaO}-t-\mathrm{Bu}(1.9 \mathrm{mg}, 0.020 \mathrm{mmol}, 2.0 \mathrm{~mol} \%)$, 
(R)-(-)-DTBM-SEGPHOS (1.2 mg, $\left.1.0 \times 10^{-3} \mathrm{mmol}, 0.05 \mathrm{~mol} \%\right), t$-BuOH $(107 \mu \mathrm{L}, 2.0 \mathrm{mmol}$, 1.0 equiv), PMHS ( $0.52 \mathrm{~mL}, 4.0 \mathrm{mmol})$, toluene $(4.0 \mathrm{~mL})$, and THF $(1.0 \mathrm{~mL})$. The reaction was quenched with $2.5 \mathrm{M}$ aqueous $\mathrm{NaOH}$ rather than $\mathrm{KF}$. The title compound (321.4 mg, 98\%) was isolated as a white crystalline solid $\left(\mathrm{mp} 42.5-43.5^{\circ} \mathrm{C}\right)$. Chiral GC analysis (Chiraldex GTA column, $\left.125^{\circ} \mathrm{C}\right)$ indicated an ee of $92.0 \% ; \mathrm{R}_{f}=0.41(20 \%$ EtOAc/hexanes $) ;{ }^{1} \mathrm{H}$ NMR $(400$ $\left.\mathrm{MHz}, \mathrm{CDCl}_{3}\right): \delta=7.22(\mathrm{bm}, 5 \mathrm{H}), 4.84(\mathrm{dd}, J=8.4 \mathrm{~Hz}, J=8.0 \mathrm{~Hz}, 1 \mathrm{H}), 3.64(\mathrm{dd}, J=6.0 \mathrm{~Hz}, J$ $=5.6 \mathrm{~Hz}, 1 \mathrm{H}), 3.46(\mathrm{dd}, J=6.0 \mathrm{~Hz}, J=5.6 \mathrm{~Hz}, 1 \mathrm{H}), 2.13(\mathrm{dt}, J=8.4 \mathrm{~Hz}, J=6.0 \mathrm{~Hz}, 1 \mathrm{H}), 2.02$ (bs, $1 \mathrm{H}), 1.98(\mathrm{dt}, J=8.0 \mathrm{~Hz}, J=6.0 \mathrm{~Hz}, 1 \mathrm{H}) .{ }^{13} \mathrm{C} \mathrm{NMR}\left(400 \mathrm{MHz}, \mathrm{CDCl}_{3}\right): \delta=143.84$, 128.81, 128.57, 128.07, 126.15, 125.95, 71.41, 41.91, 41.55. HRMS (ESI+TOF): $\mathrm{m} / \mathrm{z}$ calcd for $\mathrm{C}_{9} \mathrm{H}_{11} \mathrm{ClO} 170.0498$, found 170.0492 .

(R)-2-Phenyl-1-(thiazol-2-yl)ethanol (12; Table 1, entry f). The representative procedure was used with the following amounts of reagents: 2-phenyl-1-(thiazol-2-yl)ethanone (50.8 mg, 0.25 $\mathrm{mmol}), \mathrm{CuCl}\left(0.25 \mathrm{mg}, 2.5 \times 10^{-3} \mathrm{mmol}, 1.0 \mathrm{~mol} \%\right)$ and $\mathrm{NaO}-t-\mathrm{Bu}\left(0.24 \mathrm{mg}, 02.5 \times 10^{-3} \mathrm{mmol}\right.$,

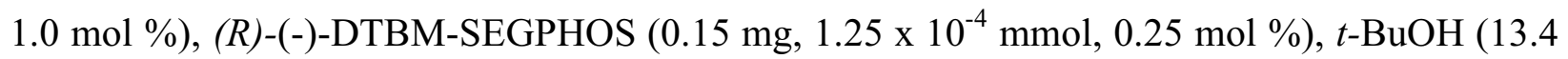
$\mu \mathrm{L}, 0.25 \mathrm{mmol}, 1.0$ equiv), PMHS (65 $\mu \mathrm{L}, 1.0 \mathrm{mmol})$, toluene $(0.30 \mathrm{~mL})$ and THF $(0.7 \mathrm{~mL})$. The reaction was quenched with $2.5 \mathrm{M}$ aqueous $\mathrm{NaOH}$ rather than $\mathrm{KF}$. The title compound (51.3 $\mathrm{mg}, 100 \%$ ) was isolated as a colorless oil. Chiral GC analysis (Chiraldex GTA column, $145^{\circ} \mathrm{C}$ ) indicated an ee of $89.3 \% ; \mathrm{R}_{f}=0.21(20 \%$ EtOAc/hexanes $) ;{ }^{1} \mathrm{H}$ NMR $\left(400 \mathrm{MHz}, \mathrm{CDCl}_{3}\right): \delta=$ $7.76(\mathrm{~s}, 1 \mathrm{H}), 7.23-7.34(\mathrm{bm}, 7 \mathrm{H}), 5.22(\mathrm{q}, J=4.0 \mathrm{~Hz}, J=8.8 \mathrm{~Hz}, 1 \mathrm{H}), 3.375(\mathrm{dd}, J=4.0 \mathrm{~Hz}, J=$ $13.6 \mathrm{~Hz}, 1 \mathrm{H}), 3.10(\mathrm{dd}, J=8.8 \mathrm{~Hz}, J=13.6 \mathrm{~Hz}, 1 \mathrm{H}), 3.05(\mathrm{bs}, 1 \mathrm{H}) ;{ }^{13} \mathrm{C} \mathrm{NMR}(400 \mathrm{MHz}$, $\left.\mathrm{CDCl}_{3}\right): \delta=174.59,142.56,127.02,129.88,128.86,127.17,119.21,72.94,44.74$. HRMS (ESI+/TOF): $m / z$ calcd for $\mathrm{C}_{11} \mathrm{H}_{11} \mathrm{NOS}(\mathrm{M}+\mathrm{H})^{+} 206.0634$, found 206.0642 . 
(S)-1-(2,4-Dichlorophenyl)-2-(1H-imidazol-1-yl)ethanol (14; Table 1, entry g). The representative procedure was used with the following amounts of reagents: 1-(2,4dichlorophenyl)-2-(1H-imidazol-1-yl)ethanone (255 mg, $1.0 \mathrm{mmol}), \mathrm{CuCl}(2.0 \mathrm{mg}, 0.02 \mathrm{mmol}$, $2.0 \mathrm{~mol} \mathrm{\%}$ ) and $\mathrm{NaO}-t-\mathrm{Bu}(1.9 \mathrm{mg}, 0.02 \mathrm{mmol}, 2.0 \mathrm{~mol} \%), R-(-)-D T B M-S E G P H O S ~(2.9 \mathrm{mg}$, $\left.2.5 \times 10^{-4} \mathrm{mmol}, 0.25 \mathrm{~mol} \%\right), t$-BuOH (90 $\mu \mathrm{L}, 1.0 \mathrm{mmol}, 1.0$ equiv), PMHS (130 $\mu \mathrm{L}, 2.0$ $\mathrm{mmol})$, toluene $(2.0 \mathrm{~mL})$ and THF $(1.0 \mathrm{~mL})$. The reaction was quenched with $2.5 \mathrm{M}$ aqueous $\mathrm{NaOH}$. The title compound (231.4 mg, 90\%) was isolated as a colorless oil. Comparison of spectral data with known literature values confirmed the identity of the product. ${ }^{5}$ Chiral GC analysis (Chiraldex GTA column, $155^{\circ} \mathrm{C}$ ) indicated an ee of $86.1 \%$.

\footnotetext{
${ }^{1}$ Gutman, A. L.; Zuobi, K; Bravdo, T. J. Org. Chem. 1990, 55, 3546.

${ }^{2}$ Wishka, D. G.; Graber, D. R.; Seest, E. P.; Lester, A.; Dolak, F. H.; Watt, W.; Morris, J. J. Org. Chem. 1998, 63, 7851.

${ }^{3}$ Tanaka, K.; Katsurada, M.; Ohno, F.; Shiga, Y.; Oda, M.; Miyagi, M.; Takehara, J.; Okano, K. J. Org. Chem. $\mathbf{2 0 0 0}, 65,432$.

${ }^{4}$ Brands, K. M. J.; Payack, J. F.; Rosen, J. D.; Nelson, T. D.; Candelario, A.; Huffman, M. A. et al. J. Am. Chem. Soc. 2003, 125, 2129.

${ }^{5}$ Lennon, I. C.; Ramsden, J. A. Org. Process Res. Dev. 2005, 9, 110 .
} 